\title{
Designing for Usability in 3D Virtual Environments
}

\author{
William Sawyerr \\ Anglia Ruskin University \\ Cambridge, United Kingdom \\ william.sawyerr@anglia.ac.uk
}

\author{
Mike Hobbs \\ Anglia Ruskin University \\ Cambridge, United Kingdom \\ mike.hobbs@anglia.ac.uk
}

\begin{abstract}
This paper is about designing 3D applications and the software design process in desktop virtual environments. Here, we introduce parts of our work on a design-focused approach to usability engineering and software development for 3D desktop virtual environments. We begin by discussing some of the current limitations in virtual environment research. We then introduce alternate perspectives for improving the usability of virtual environment applications. We highlight the need for a software development approach to designing virtual environment applications and provide some arguments for this. Finally, we conclude by providing a summary of our work-in-progress solution.
\end{abstract}

Index Terms-Usability, usability engineering, design languages, interactive applications, elderly users, virtual environments, virtual worlds.

\section{INTRODUCTION}

This paper introduces parts of our work on a design-focused approach to usability engineering and software development for 3D desktop virtual environments. The research presented here is about designing $3 \mathrm{D}$ applications and the software design process in desktop virtual environments. Our ultimate goal is to create a means to bolster the condition of virtual environment applications (i.e., robustness, reliability, etc.) in-between requirements engineering activities and later stages of development (i.e., implementation, testing, deployment, etc.). By taking this approach, we hope to improve the usability of interactive 3D applications (i.e., ones that use scripts or programs to allow them to accept and respond to input from humans) as well as the chances of successfully deploying these applications for practical use in the real world.

Our research problem stems from much larger issues in virtual environment research. These are usability of 3D virtual environment user interfaces [1], [2], and designing 3D virtual environments for limited access groups, using the elderly as an example [3], [4]. Within these two areas of inquiry, there have been several challenges that have influenced our work to focus more on design. One of those challenges is that there are minor limitations to work that is (and has) been conducted on the usability of 3D virtual environment user interfaces and designing 3D virtual environments for the elderly. These limitations to past and present work fuel some serious misunderstandings about the nature of virtual environment research. They are also a barrier to the adoption of virtual environments for practical uses in the real world. Therefore, if we expect progress and the full potential of virtual environments to be realised, then these limitations must be addressed.

\section{LIMITATIONS IN VIRTUAL ENVIRONMENT RESEARCH}

The status of usability of virtual environments gives us a very good example and a starting point to highlight some of the limitations in virtual environment research. Given how long virtual environment researchers have been focusing on usability as a research topic, there is little within the literature to suggest that virtual environments are currently a usable product. In other words, besides reports on the potential of virtual environments [5], [6], and the move towards serious uses or applications of virtual environments [7], [8], there are few existing claims that virtual environments are in fact usable. Instead, empirical and other observational data point to the contrary [9], [10]. Virtual environments are still plagued with problems and usability (i.e., poor usability) has been implicated as a reason for many of these problems. Poor usability has also been cited as a reason for the slow rate of adoption of these platforms for serious uses [11], [12].

The principles of usability are concerned with ease of use, learnability, and are connected to the usefulness of a product [13]. The outcome of a usability study is generally expected to be some recommendations on how to improve the product and how to make it easier and more enjoyable to use. However, both usability studies and what we do with the outcome of these studies is currently a complicated matter for virtual environment research. This is because there are very few existing standards (or metrics) that can be specifically used to evaluate the usability of virtual environments. Likewise, there are very few guidelines that can be specifically used for improving the usability of virtual environments. Depending on the application area and context of use, these existing standards and guidelines may not work very well. In such cases, the resort has been to use ad-hoc methods [14], [15], that now remain scattered across virtual environment research and are of little use beyond a single application area or context of use.

For the most part, researchers and designers have approached virtual environments as a single dimension (i.e., the virtual environment is mostly treated as the platform and also as the application). However, in approaching the design of interactive $3 \mathrm{D}$ applications from a software development perspective, we find it necessary to organise virtual environments into multiple dimensions. 


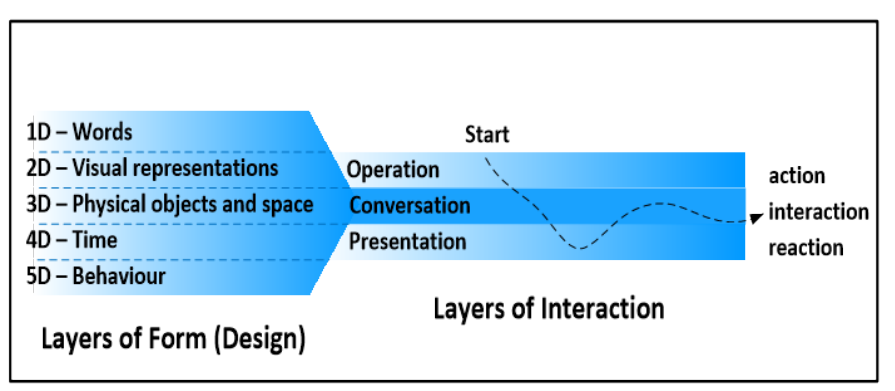

Fig. 1. Abstraction Layers of Virtual Environment Form (Design) and Interaction.

Fig. 1 above is an adaptation of the five dimensions of interaction design in software development [16], [17]. These dimensions are used as layers of abstraction in order to align application development in virtual environments with traditional software development for other platforms. Furthermore, by organising virtual environments into logical layers of abstraction, we are able to approach usability in virtual environments more easily by focusing on specific problem areas.

In previous research, we have suggested that there needs to be an abstraction between virtual environments and the applications built in them, including a respective abstraction of the user interfaces [18]. The user interface of an application within a virtual environment is different from the user interface of the virtual environment itself. In the context of 3D desktop virtual environments, an application is a 3D object (or collection of 3D objects) along with any embedded scripts (interactive or non-interactive) that provide some form of service within the virtual environment. On the other hand, the virtual environment is the physical 3D space that contains the application. 'The Chaotic Science Lab', which aims to provide support for trainee science teachers, gives us an example of a production-type application in a virtual environment [19].

The user interfaces of both the application and the virtual environment may present different types of usability problems for users. For example, problems faced with opening a virtual door are different from problems faced with setting camera controls on the virtual environment viewer. Likewise, some of the error messages that may come from the virtual environment platform can be very cryptic for normal non-technical users to understand whilst designers of virtual environment applications have much more control in creating user-friendly error messages.

Researchers and designers have spent very little time addressing the limitations in virtual environment research that we have discussed. Despite all the advances in virtual environment technology, these limitations make designing realworld applications a challenge and possibly premature. This is especially true for certain user groups such as the elderly who are known to have particular needs that are different from the generic user base of virtual environments [20], [21], and technology in general [22], [23].

\section{DESIGNING FOR USABILITY: AN ALTERNATIVE APPROACH TO IMPROVING USABILITY}

Usability studies in virtual environments is still in its infancy and a body of knowledge is being developed that will drive future research. Usability evaluation methods are still in the process of being created and validated [24], [25], [26], and design methodologies are also still in the process of being developed [27], [28], [29]. Besides this, a few other problems demand building up other areas of virtual environment research before focusing on creating real-world applications. Usability is a very wide area of research with several other dimensions such as usability frameworks, usability theories, usability models etc. However, our findings suggest that research on usability is biased towards usability evaluations, leaving other areas of research less developed. The outcome is that it is quite difficult to design production-type (or real-world) applications without any existing guiding principles or some methodology that guides the process. Therefore, our research addresses this gap by shifting focus towards designing virtual environment applications.

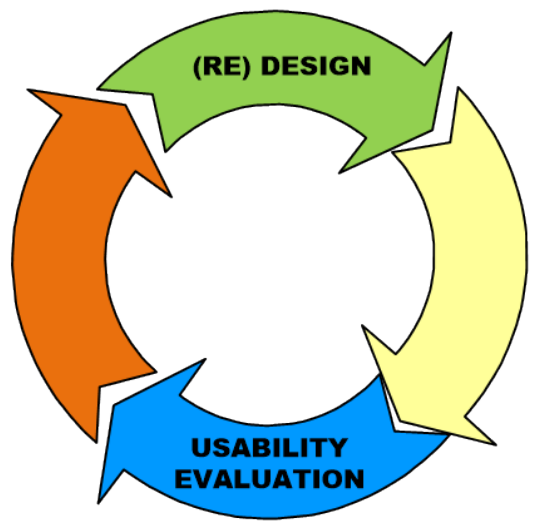

Fig. 2. The Usability Evaluation Cycle (the current model used in virtual environment research).

By focusing on design, we can build applications that are more robust and rely less on usability evaluations after production. Our approach does not undermine the importance of usability evaluations. Nevertheless, there are advantages for focusing on design and less on evaluations. For example, Fig. 2 above shows the current model for managing usability in virtual environment research. Typically, after a virtual environment and its applications are deployed for use, evaluations on the usability of the applications can begin. Since there are no evaluation methods that exist specifically for use in evaluating virtual environments or the applications that are built in them, existing methods are either used or ad-hoc methods are created to perform the evaluation. As we explained earlier, existing methods may not work so well and ad-hoc methods are rarely reusable. Nonetheless, whatever the choice is between existing or ad-hoc methods, usability evaluations are meant to reveal problems that then become recommendations for redesign. The evaluation process is iterative and continues until most of the problems in the 
application or the virtual environment have been contained. Because the current model of usability evaluations is highly iterative, it is costly and requires lots of time and human capital. The benefit of focusing on designing usable applications as opposed to iteratively redesigning for usability is that we can reduce the overhead expenses related to usability evaluations. If applications in virtual environments are designed very well, it will also reduce the possibility that users (especially first-time users) will become frustrated with using these applications or virtual environments in general.

\section{SOFTWARE DEVELOPMENT FOR VIRTUAL ENVIRONMENTS}

Our observation is that what has been ordinarily referred to as 'designing virtual environments' is in fact the implementation and subsequent deployment of these platforms [30], [31], [32]. Within our design research, we are addressing other things such as formalising the characteristics of virtual environment applications and the user interface. For now, we intend to use a generic software development approach in order to have a point of reference that guides the development process of virtual environment applications. We do this because we believe that the development of virtual environment applications should be formally treated as software development is done for many other platforms such as mobiles, PCs, etc. It should be systematic. A new methodology does not necessarily need to be created for this task. For example, we will be using the unmodified waterfall model for software development by Royce [33]. Royce's waterfall model is made up of the following software development stages:

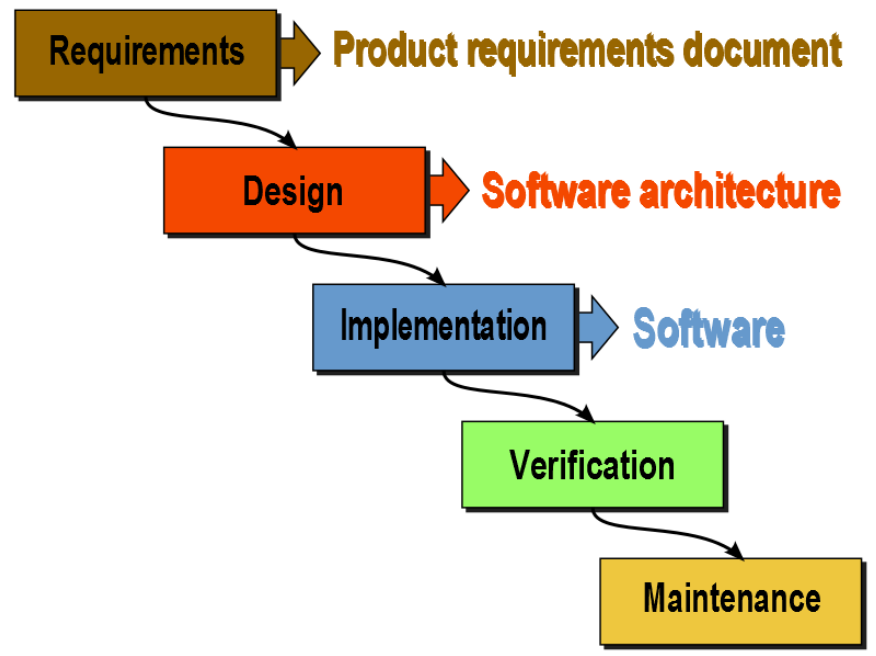

Fig. 3. The Generic, Unmodified Waterfall Software Development Model.

We acknowledge that the waterfall model in Fig. 3 above has been found to have shortcomings for modern software development. However, practice has also shown that the waterfall model is well-suited for design-focused software development projects, is efficient and economical, and can supplement and thereby coexist with other approaches [34]. We also have other reasons for choosing the waterfall model. If our research is about designing virtual environment applications in order to improve their condition, then it is helpful to ground this activity in software development. It is also helpful to show that designing virtual environment applications does have a place in (and is a necessary part of) software development. Therefore, we use the waterfall model as a general pointer for our activities. Designing virtual environment applications is obviously part of the design stage of the waterfall software development model shown in Fig. 3. Usability evaluations typically take place anywhere after the implementation stage.

\section{CONCLUSIONS AND FUTURE WORK}

In this paper, we have discussed some of the limitations that affect virtual environment research. We also suggested that there are problems with the current model of managing usability in virtual environment research. In particular, we discuss the bias towards usability evaluations and some of the implications for that. We have suggested focusing more on design as an alternative approach to improving the usability of virtual environment applications. We base our ideas on the premise that putting more effort on properly designing virtual environment applications will reduce the usability problems that are normally found after deployment.

Our current research goal is to create a design language that will be used for virtual environment applications. We are working on a meta-artefact, a tool, which will be used to design applications in virtual environments. The development of the design language draws from theories in linguistics and the philosophy of language. The design language will specifically derive from speech act theory, which relates to utterances that have performative function in language and communication [35], [36]. We intend to use speech act theory in order to show how language can be used to characterise design in virtual environments. We complement this with the language-action perspective (LAP), which derives from speech act theory and was intended to be applicable to the design of computer systems [37].

We will firstly use the theories above to document and validate the structure of the design language before progressing to an actual characterisation of it. The characterisation of the design language will be based on an architectural model for a design language in text-based (1D) virtual environments [38]. The model for the design language evolves from the idea that virtual environments are organised in a linguistic manner, and therefore, language can be used as a tool to perform events or actions on them [39]. We aim to produce a final product in natural language form.

\section{ACKNOWLEDGMENT}

We would like to thank Elaine Brown and Dr. Silvia Cirstea at Anglia Ruskin University, for their helpful insights and for sharing their expertise with us. We would also like to thank Professor Marcian Cirstea at Anglia Ruskin University, for his support.

\section{REFERENCES}

[1] K. Kaur, "Designing virtual environments for usability," INTERACT. New York, pp. 636-639, July 1997. 
[2] A. G. Sutcliffe and K. D. Kaur, "Evaluating the usability of virtual reality user interfaces," Behav. Inform. Technol. Vol. 19, pp. 415-426, November 2010.

[3] L. M. Camarinha-Matos and H. Afsarmanesh, "Design of a virtual community infrastructure for elderly care," in Collaborative Business Ecosystems and Virtual Enterprises, L. M. Camarinha-Matos, ed. New York: Springer US, 2002, pp. 439-450.

[4] S. N. W. Shamsuddin, V. Lesk, and H. Ugail, "Virtual environment design guidelines for elderly people in early detection of dementia," WASET. Vol. 5, pp. 11-23, 2011.

[5] W. S. Bainbridge, "The scientific research potential of virtual worlds," Science. Washington, D.C., vol. 317, pp. 472-476.

[6] T. Kohler, J. Fueller, K. Matzler, and D. Stieger, "Co-creation in virtual worlds: the design of the user experience," Mis. Quart. Minneapolis, vol. 35, pp. 773-788, September 2011.

[7] C. W. Thompson, "Next-generation virtual worlds: architecture, status, and directions," IEEE Internet Comput. Vol. 15, pp. 6065, February 2011.

[8] W. Scacchi, The Future of Research in Computer Games and Virtual World Environments. Irvine, CA: University of California, 2012.

[9] T. Barker, "Usability and affordances for teaching and learning in second life," in Handbook of Research on Practices and Outcomes in Virtual Worlds and Environments, H. Yang and S. Yeun, Eds. Hershey, PA: IGI Global, 2012, pp. 462-478.

[10] H. Lu, T. Brockmann and S. Stieglitz, "Usability of virtual worlds," in Design, User Experience, and Usability. Design Philosophy, Methods, and Tools, A. Marcus, ed. Berlin: Springer Berlin Heidelberg, 2013, pp. 340-348.

[11] A. Luse, B. Mennecke and J. Triplett, "The changing nature of user attitudes toward virtual world technology: a longitudinal study," Science Direct. Vol. 29, pp. 1122-1132, November 2012.

[12] T. E. Yoon and J. F. George, "Why aren't organizations adopting virtual worlds?," Science Direct. Vol. 29, pp. 772-790, May 2013.

[13] J. Nielsen, "Usability 101: Introduction to usability," unpublished.

[14] A. D. Lucia, R. Francese, I. Passero and G. Tortora, "Development and evaluation of a virtual campus on Second Life: the case of SecondDMI," Science Direct. Vol. 52, pp. 220233, January 2009.

[15] S. D. Freitas, G. Rebolledo-Mendez, F. Liarokapis, G. Magoulas and A. Poulovassilis, "Learning as immersive experiences: using the four-dimensional framework for designing and evaluating immersive learning experiences in a virtual world," Brit. J. Educ. Technol. Vol. 41, pp. 69-85, January 2010.

[16] B. Moggridge, Designing Interactions. Cambridge, MA: The MIT Press, 2007.

[17] K. Silver, "What puts the design in interaction design," unpublished.

[18] W. Sawyerr, E. Brown and M. Hobbs, "Using a hybrid method to evaluate the usability of a virtual environment user interface," IJITCS. Kathmandu, vol. 8, pp. 66-74, April 2013.

[19] D. Holley, M. Hobbs, P. Howlett and W. Sawyerr, "“The chaotic science lab': supporting trainee Science teachers - a cross departmental project," Networks. Cambridge, pp. 51-57, January 2013.
[20] P. Jansen, A. Schmelter and M. Heil, "Spatial knowledge acquisition in younger and elderly adults: a study in a virtual environment," Exp. Psychol. Vol. 57, pp. 54-60, 2010.

[21] P. Siriaraya and C. S. Ang, "Age differences in the perception of social presence in the use of 3D virtual worlds for social interaction,” Interact. Comput. Vol. 24, pp. 280-291, 2012.

[22] S. E. Lindley, A. Sellen and R. Harper, "Bridging the gap between grandparents and teenagers: lightweight vs. heavyweight contact," CHI EA. April 2009.

[23] S. E. Lindley, "Shades of lightweight: supporting crossgenerational communication through home messaging," UAIS. Vol. 11, pp. 31-43, March 2012.

[24] C. Rusu, R. Munoz, S. Roncagliolo, S. Rudloff, V. Rusu and A. Figueroa, "Usability heuristics for virtual worlds," AFIN. Nice, pp. 16-19, August 2011.

[25] B. Koehne and D. F. Redmiles, "Envisioning distributed usability evaluation through a virtual world platform," IEEE CHASE. pp. 73-75, June 2012.

[26] R. Munoz and V. Chalegre, "Defining virtual worlds usability heuristics," IEEE ITNG. pp. 690-695, April 2012.

[27] S. Tanney, P. Schwartz, S. Yen, L. Shen and T. Furness, "A design method for virtual environments using narrative and pattern languages," HITL Technical Report. University of Washington, 1998.

[28] T. L. Ross and R. D. Cornell, "Towards an experimental methodology of virtual world research," IEEE CS. pp. 143-150, March 2010.

[29] B. Bauer, "Considerations and methodology for designing a virtual world: solution for a large corporation," in Gaming and Simulations: Concepts, Methodologies, Tools and Applications. Hershey, PA: IGI Global, 2011, pp. 931-408.

[30] K. Kaur, "Designing virtual environments for usability," unpublished.

[31] R. Hamalainen, "Designing and evaluating collaboration in a virtual game environment for vocational learning," Science Direct. Vol. 50, pp. 98-109, January 2008.

[32] D. C. Short, "Designing a 3D virtual HRD environment from a scholar-practitioner perspective," ADHR. Vol. 15, pp. 270-283, August 2013.

[33] W. W. Royce, "Managing the development of large software systems," IEEE WESCON, pp. 1-9, August 1970.

[34] P. Kruchten, "Software architecture and agile software development - a clash of two cultures?," IEEE ICSE, pp. 497498, May 2010.

[35] J. L. Austin, How to do Things with Words, 2nd ed., Oxford: Oxford Press, 1975.

[36] J. R. Searle, Speech Acts: An Essay in the Philosophy of Language. Cambridge: Cambridge University Press, 1969.

[37] T. Winograd and F. Flores, Understanding Computers and Cognition: A New Foundation for Design. Reading, MA: Addison-Wesley, 1987.

[38] A. Cigognani, "Designing with words: a model for a design language in a MOO" CGIT. Orlando, pp. 19-24, July 1998.

[39] A. Cicognani, "On the linguistic nature of cyberspace and virtual communities," VR. Berlin, vol. 3, pp. 16-24, 1998. 